\title{
Polio eradication initiative in Africa: influence on other infectious disease surveillance development Peter Nsubuga*1, Sharon McDonnell ${ }^{1}$, Bradley Perkins ${ }^{2}$, Roland Sutter ${ }^{3}$, Linda Quick ${ }^{3}$, Mark White ${ }^{1}$, Stephen Cochi ${ }^{3}$ and Mac Otten ${ }^{3,4}$
}

\begin{abstract}
Address: ${ }^{1}$ Division of International Health, Epidemiology Program Office, Centers for Disease Control and Prevention, Atlanta, Georgia, USA 2Division of Bacterial and Mycotic Diseases, National Center of Infectious Diseases, Centers for Disease Control and Prevention, Atlanta, Georgia, USA, ${ }^{3}$ Vaccine Preventable Disease Eradication Division, National Immunization Program, Centers for Disease Control and Prevention, Atlanta, Georgia, USA and ${ }^{4}$ Regional Office for Africa, World Health Organization, Harare, Zimbabwe

Email: Peter Nsubuga* - pnsubuga@cdc.gov; Sharon McDonnell - sem0@cdc.gov; Bradley Perkins - bap4@cdc.gov; Roland Sutter - rsutter@cdc.gov; Linda Quick - maq2@cdc.gov; Mark White - mhw1@cdc.gov; Stephen Cochi - scochi@cdc.gov; Mac Otten - ottenm@whoafr.org

* Corresponding author
\end{abstract}

Published: 27 December 2002

BMC Public Health 2002, 2:27
Received: 17 September 2002

Accepted: 27 December 2002

This article is available from: http://www.biomedcentral.com/I47I-2458/2/27

(C) 2002 Nsubuga et al; licensee BioMed Central Ltd. This is an Open Access article: verbatim copying and redistribution of this article are permitted in all media for any purpose, provided this notice is preserved along with the article's original URL.

\begin{abstract}
Background: The World Health Organization (WHO) and partners are collaborating to eradicate poliomyelitis. To monitor progress, countries perform surveillance for acute flaccid paralysis (AFP). The WHO African Regional Office (WHO-AFRO) and the U.S Centers for Disease Control and Prevention are also involved in strengthening infectious disease surveillance and response in Africa. We assessed whether polio-eradication initiative resources are used in the surveillance for and response to other infectious diseases in Africa.
\end{abstract}

Methods: During October 1999-March 2000, we developed and administered a survey questionnaire to at least one key informant from the 38 countries that regularly report on polio activities to WHO. The key informants included WHO-AFRO staff assigned to the countries and Ministry of Health personnel.

Results: We obtained responses from $32(84 \%)$ of the 38 countries. Thirty-one $(97 \%)$ of the 32 countries had designated surveillance officers for AFP surveillance, and $25(78 \%)$ used the AFP resources for the surveillance and response to other infectious diseases. In 28 (87\%) countries, AFP program staff combined detection for AFP and other infectious diseases. Fourteen countries (44\%) had used the AFP laboratory specimen transportation system to transport specimens to confirm other infectious disease outbreaks. The majority of the countries that performed AFP surveillance adequately (i.e., non polio AFP rate $=1 / 100,000$ children aged $<15$ years) in 1999 had added I-5 diseases to their AFP surveillance program.

Conclusions: Despite concerns regarding the targeted nature of AFP surveillance, it is partially integrated into existing surveillance and response systems in multiple African countries. Resources provided for polio eradication should be used to improve surveillance for and response to other priority infectious diseases in Africa. 


\section{Background}

The polio-eradication initiative has led to the largest influx of public health resources into Africa since the smallpox-eradication campaign, comprising both human resources and infrastructure investment [1,2]. Public health professionals have debated the merits and demerits of the polio-eradication initiative, regarding the priorities of developing countries. Supporters of the initiative have reported on the high benefit-cost ratio of eradication $[1,2]$. Among the demerits cited is that polio has a lower public health importance as compared to other infectious diseases - many of them epidemic prone - in poor countries [1-3]. An investigation of the impact of the polioeradication initiative on the status of funding for routine immunization revealed that the amount of funding for routine immunization activities has not increased over the years and that whether eradication funding will be available for other public health interventions when polio is eradicated is unclear [4]. Anecdotally reported merits and benefits of the polio-eradication initiative have included increased national enthusiasm and funding for Expanded Programs on Immunization, enhanced surveillance capacity for other diseases, strengthened public health laboratory capacity, and improved epidemiologic skills [2]. We report the results of a survey regarding the impact of the polio-eradication initiative on the surveillance for other infectious diseases in Africa.

In 1989, the African Regional Office of the World Health Organization (WHO-AFRO) adopted the global goal of eradicating poliomyelitis by the year 2000, and in 1995 , member countries initiated specific polio-eradication strategies including acute flaccid paralysis (AFP) surveillance. Resources are deployed primarily through surveillance officers, development of a functional regional laboratory network, logistics, cold-chains, communications, and transportation [5-7]. To ensure that the polio eradication strategies were efficiently and effectively implemented, countries were divided into five epidemiological blocks on the basis of geographic proximity, similarity in infrastructure, and program needs. These country groupings included central, eastern, southern, and western epidemiological blocks and Countries in Special Circumstances (i.e., Angola, Democratic Republic of the Congo, Ethiopia and Nigeria) [5]. By 1999, 38 countries regularly reported polio/AFP surveillance data to WHOAFRO.

In September 1998, to share resources, and improve efficiency, WHO-AFRO adopted the Integrated Disease Surveillance and Response (IDSR) strategy, which aims to improve surveillance and response by integrating infectious disease surveillance programs $[8,9]$. The IDSR strategy is based on core activities and support functions that are required to perform infectious disease surveillance, ep- idemic preparedness, and response $[1,10,11]$. Core activities include case-patient detection, registration, and confirmation; reporting, analysis, use, and feedback of data; and epidemic preparedness and response (e.g., outbreak investigations, contact tracing, and public health interventions). Support functions include coordination, supervision or performance evaluation, training, and resource-provision for infrastructure, including communication. The Centers for Disease Control and Prevention (U. S. Department of Health and Human Services) (CDC) provides technical and financial support to WHO-AFRO for both IDSR and the polio-eradication initiative, along with the U. S. Agency for International Development and other partners.

\section{Methods}

We performed a survey to determine the impact of AFP surveillance on the surveillance for and response to other infectious diseases. The objectives of the survey included describing the characteristics of AFP surveillance programs in WHO-AFRO, how surveillance activities for AFP and other infectious diseases have been combined, the contribution of AFP surveillance to the surveillance of other infectious diseases, and the effect of adding surveillance and response to other infectious diseases on the performance of AFP surveillance. We also used the experience of AFP surveillance programs to obtain a description of the constraints to strengthening infectious disease surveillance in Africa. We targeted the 38 countries that regularly report AFP surveillance and polio eradication activities to World Health Organization (WHO).

We developed, pilot-tested, and translated into French a survey questionnaire. The questionnaire was administered to at least one key informant per country by telephone, electronic mail, and in person during December 1999-April 2000. The key informants included Ministry of Health officials and WHO country assignees. Non-polio AFP rates are used as an indicator of the sensitivity of AFP surveillance programs. A sensitive AFP surveillance program should be able to detect a background rate of $>1$ case of non-polio AFP per 100,000 children aged $<15$ years in any geographic area (province, country, region etc.) per year. Non-polio AFP rates are reported monthly to WHO and compiled annually. We abstracted data regarding non-polio AFP rates from the WHO website [12]. Data accruing from this survey were entered and analyzed using Epi Info version 6.04 [13].

\section{Results}

We obtained data from 32 (84\%) of the 38 countries that regularly report on polio/AFP surveillance activities in Africa to $\mathrm{WHO}$ (Figure 1). The highest response rates were from the southern epidemiological blocks (all nine countries) and the Countries in Special Circumstances (all four 


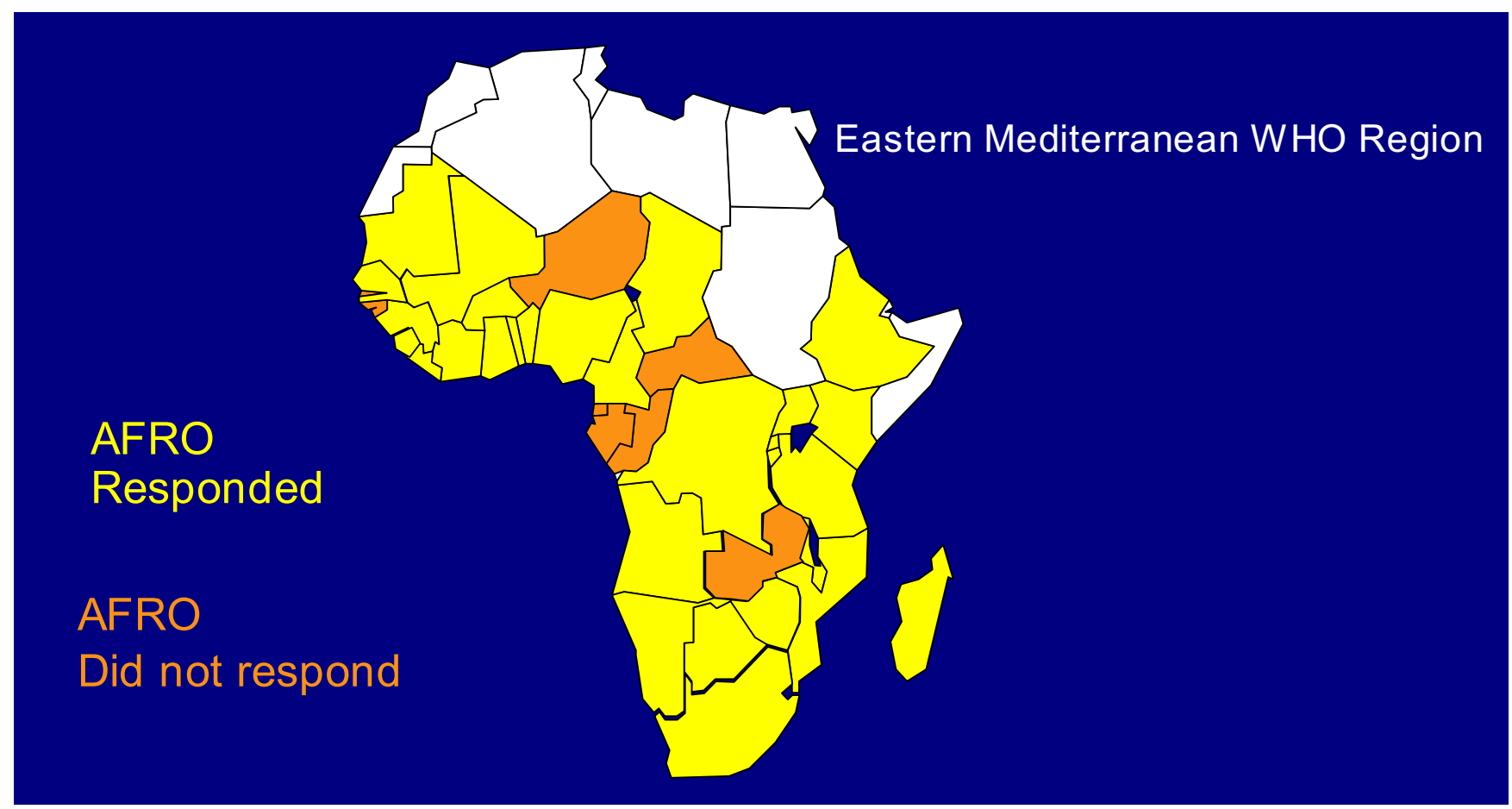

Figure I

African Region WHO (AFRO) Countries Surveyed - 2000

countries). The lowest response rate was from the central epidemiological block where only two of the four countries responded.

Thirty-one $(97 \%)$ of the 32 countries had designated surveillance officers working on AFP surveillance, with a median number of 10 per country. Twenty-seven $(84 \%)$ of the 32 countries had an annual AFP surveillance budget ranging from $\$ 10,000$ to $\$ 1.8$ million (median: $\$ 125,000)$. The majority AFP surveillance programs (26 [81\%]) had $>1$ dedicated vehicles. In the majority of countries (27 [84\%]), the AFP surveillance program was started after the general infectious disease surveillance program.

Detection for other infectious diseases was combined with AFP detection in 28 (90\%) countries (Table 1). In 28 $(90 \%)$ countries, staff from the AFP program took responsibility for informing clinicians and other health practitioners regarding other infectious diseases while conducting training in AFP surveillance. Fourteen countries (44\%) had used the AFP laboratory specimen transportation system to transport specimens to laboratories for confirmation other infectious disease outbreaks.
Fifteen $(47 \%)$ of the 32 countries performed AFP surveillance adequately (i.e., non polio AFP rate $=1 / 100,000$ children aged <15 years) at the end of 1999 (Figure 2). Eleven $(73 \%)$ of the 15 countries that performed AFP surveillance adequately had also added 2-5 diseases onto their AFP surveillance program. Both countries that added $>5$ diseases onto their AFP surveillance program performed AFP surveillance inadequately.

A total of 25 (78\%) of the 32 countries had combined AFP surveillance with surveillance for other infectious diseases. The most common diseases added to surveillance for AFP were other childhood vaccine-preventable diseases: measles in 24 (96\%) and neonatal tetanus in 22 (88\%). The other diseases added to the AFP surveillance programs tended to be epidemic-prone diseases (e.g., cholera in 17 [68\%], meningitis in 16 [64\%], or yellow fever in 11 [44\%]), and depended on the epidemiological patterns in the responding countries.

When the respondents were asked to illustrate major contributions attributable to the AFP surveillance programs, $12(38 \%)$ described an improvement of national disease surveillance (Table 2). Other contributions cited were improved infrastructure or resources, increased awareness regarding surveillance or capacity building, and increased 


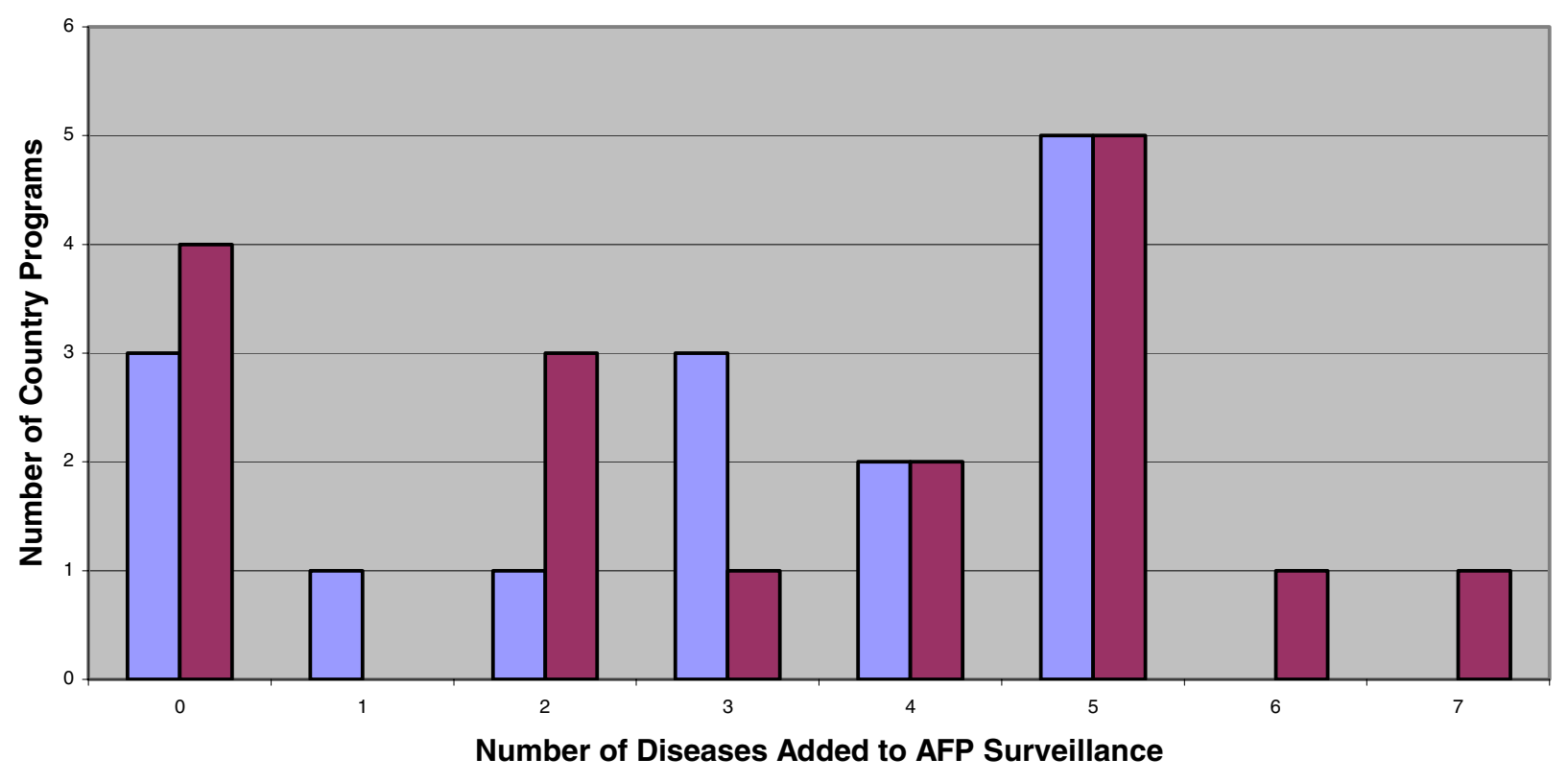

$\square$ Adequate $\square$ Inadequate

Adequate performance $=$ Non polio AFP rate $\geq 1$ per 100,000 children less than 15 years old Inadequate performance $=$ Non polio AFP rate $<1$ per 100,000 children less than 15 years old

\section{Figure 2}

Effect of integration of other diseases on the performance of acute flaccid paralysis (AFP) surveillance using I999 non polio AFP rates in 32 African countries

Table I: Integration of surveillance and response for other diseases with the acute flaccid paralysis surveillance program in 32 African countries -2000

\begin{tabular}{lll}
\hline Attribute & $\mathbf{n}$ & (\%) \\
\hline Use AFP resources for surveillance for other diseases & 26 & $(81)$ \\
Combine detection for other diseases with AFP & 28 & $(90)$ \\
Inform clinicians about other diseases when informing them about AFP & 27 & $(87)$ \\
Use AFP laboratory transportation system for other diseases & 14 & $(44)$ \\
\hline Total & 32 & \\
\hline
\end{tabular}

personnel for surveillance. Major constraints to general disease surveillance that were identified included a lack of staff to perform surveillance, a shortage or lack of funds, lack of vehicles or fuel, lack of training, and a lack of political commitment.

\section{Discussion}

Our survey revealed that, among the African countries that conducted AFP surveillance and reported to WHO in 1999, the majority had designated surveillance officers, vehicles, and annual budgets. Moreover, most of the national polio eradication programs combined the surveillance for and response to AFP with other infectious diseases. Our investigation also revealed that certain 
Table 2: Major contribution of acute flaccid paralysis surveillance to surveillance for other diseases and constraints to disease surveillance in 32 African countries - 2000

\begin{tabular}{|c|c|c|}
\hline Contribution of AFP Surveillance & $\mathbf{n}$ & (\%) \\
\hline Improvement of disease surveillance & 12 & (38) \\
\hline Improved infrastructure or resources & 7 & (22) \\
\hline Increase awareness about surveillance or capacity building & 7 & (22) \\
\hline Increased personnel for surveillance & 3 & (9) \\
\hline \multicolumn{3}{|l|}{ Constraints to Disease Surveillance } \\
\hline Lack of staff & 9 & (28) \\
\hline Lack of funds & 7 & (22) \\
\hline Lack of vehicles or fuel & 3 & (9) \\
\hline Lack of training & 2 & (6) \\
\hline Lack of political commitment & 1 & (3) \\
\hline Total & 32 & \\
\hline
\end{tabular}

countries that had prudently added other diseases to their AFP surveillance programs were also able to perform AFP surveillance adequately. However, the survey also indicated that additional staff, funds, and political commitment might be required if infectious disease surveillance and response is to improve in Africa.

AFP surveillance programs have instituted laboratory systems in countries and have formed networks between and within countries and with WHO-AFRO by providing portable computers with modems, telephone/facsimile lines, and electronic mail connections. Our survey results indicated that detection and confirmation of outbreaks of other infectious diseases has been conducted by using the resources and infrastructure of the AFP surveillance laboratory network, including the specimen transportation system. This can be further strengthened into a network of laboratories that can support the IDSR strategy. Joint information sessions for clinicians regarding polio-AFP surveillance and other diseases of public health importance within countries are already occurring and can be strengthened into a collaborative training effort for IDSR. Stronger links among epidemiologists and laboratorians are necessary to improve outbreak detection and control.

Our study determined that the majority of diseases that are integrated into AFP programs are vaccine-preventable diseases (e.g., measles and neonatal tetanus), indicating an ongoing linkage with other vaccination programs and disease-prevention activities. Other diseases included in AFP surveillance programs were epidemic-prone diseases (e.g., cholera and meningitis), reflecting the importance of epidemic response in general. Among the countries that achieved the surveillance target for AFP in 1999, the majority included 1-5 other infectious diseases in the AFP surveillance program, indicating that the judicious addition of a few diseases to a program such as AFP surveillance is feasible without adversely affecting the primary program.
Substantial fixed costs are involved in building and maintaining national surveillance and response systems and a limited number of countries can afford the cost of duplicative systems [14]. Other targeted and substantially funded disease control programs (e.g., HIV/AIDS, malaria, tuberculosis) need to consider following the example of AFP surveillance and make investments in the surveillance and response infrastructure at the country level. Many of these categorical programs desire improved timeliness and completeness of district reporting and evidence-based decision making, which can be addressed by implementing IDSR activities.

To support surveillance activities as a necessary component of disease prevention and control activities, an urgent need exists to develop a consensus core set of surveillance and response indicators that are field-tested and that can be monitored routinely in a similar manner to the polio indicators, in addition to the existing surveillance evaluation frameworks $[15,16]$. These indicators can then guide the strengthening of surveillance systems and the integration of other diseases into targeted disease-specific programs.

Contributions that were identified and attributable to the presence of AFP surveillance programs indicate that polioeradication programs have gone beyond a purely vertical approach (i.e., disease-specific) toward one that is more horizontal (i.e., systems development). Improvements of infrastructure, capacity building, and provision of personnel can be used to develop the overall surveillance system for infectious diseases as long as the categorical program policies clearly support this approach. A lack of resources (e.g., staff, funds, vehicles, or fuel) were the main constraints to infectious disease surveillance that were identified in the survey - interestingly, training was not identified as a top constraint, possibly indicating that trained personnel already exist, at least within the AFP surveillance program. Managers of categorical programs are 
often uneasy regarding entrusting others with gathering surveillance data that are crucial to targeting and evaluating their programs. Therefore, ongoing training monitoring and periodic external evaluations should provide the quality assurance and credibility that integrated surveillance and response programs will need to reassure managers that they are basing decisions on reliable information.

This survey had a few limitations and we were unable to obtain responses from all the targeted countries. In certain countries, we could not contact possible respondents because of difficulties in communication, which could have led to introduction of bias in the survey because the nonrespondents might have had substantially different answers to our questions than the respondents. Another possible limitation was that respondents were reporting on themselves and could have lacked objectivity additionally, the future employment and/or career development of the respondents may likely depend on their capacity to support other control programs and this may have introduced a bias in answering the questionnaire, although there is no way to determine this for certain. Further, the tool that we used did not have questions on the acceptability of AFP surveillance and the feasibility of IDSR and we also did not evaluate the cost of IDSR or the cost to maintain and sustain the infrastructure of the polio eradication initiative after polio is eradicated. We determined the performance of the AFP surveillance programs solely by non polio AFP rates because of the lack of widely used surveillance and response indicators at the time of the survey, however WHO-AFRO and CDC have recently begun work on a list of core indicators that will help monitor and evaluate the implementation of the IDSR process.

The findings of this survey have important implications for WHO-AFRO's initiative to improve surveillance, epidemic preparedness, and response in the African region. First, polio-eradication initiative staff, financial resources, and infrastructure can be used as one strategy to build IDSR in Africa. Because additional funds are needed for surveillance now and will be needed after polio is eradicated, other disease-specific programs, especially those focusing on epidemic prone diseases like malaria, might consider investing in general infectious disease surveillance following the polio example. Second, as surveillance and response capacity are developed in Africa, adding new diseases to existing or new surveillance systems should be on the basis of indicators of the surveillance system's capacity not to overload the surveillance system. Finally, the cadre of new people trained in surveillance by the polioeradication initiative should be used for IDSR, and career paths should be provided for them as one lasting legacy of the poliomyelitis-eradication campaign in Africa.

\section{Competing Interests}

None declared

\section{Author Contribution}

PN conceived and carried out the study and drafted the manuscript. SM conceived and carried out the study. BP, $\mathrm{RS}, \mathrm{LQ}, \mathrm{MW}, \mathrm{SC}$ and $\mathrm{MO}$ participated in the design and coordination of the study. All authors read and approved the final manuscript.

\section{References}

I. Taylor CE, Cutts T and Taylor ME Ethical dilemmas in current planning for polio eradication. Am J Public Health 1997, 87:922-5

2. Sutter RW and Cochi SL Ethical dilemmas in worldwide polio eradication programs [Comments]. Am J Public Health 1997, 87:913-6

3. Lee JW, Melgaard B, Hull HF, Barakamfitiye D and Okwo-Bele JM Ethical dilemmas in polio eradication. Am J Public Health 1998, 88: $130-2$

4. Levin A, Jorissen J, Linkins J and McArthur C Impact of polio eradication initiative on donor contributions to routine immunization. Special Initiative Report No. 36. Bethesda, MD: Partnerships for Health Reform Project, Abt Associates Inc 200I,

5. Okwo-Bele JM, Lobanov A, Biellik RJ, Birmingham ME, Pierre $L$ and Tomori O Overview of poliomyelitis in the African Region and current regional plan of action. J Infect Dis 1997, I 75(Suppl I):SI0-5

6. Centers for Disease Control and Prevention Progress toward polio eradication Africa, 1996. MMWR 1997, 46:32I-4

7. World Health Organization, Regional Committee for Africa Expanded program on immunization: Disease control goals; the countdown has started. Brazzaville: World Health Organization, Regional Office for Africa, resolution AFR/RC45/R5 1995,

8. World Health Organization, Regional Office for Africa Integrated disease surveillance strategy, a regional strategy for communicable diseases 1999-2003. Harare: World Health Organization Regional Office for Africa 1999 ,

9. World Health Organization: Regional Office for Africa Regional Committee resolution AFR/RC48/R2 1998,

10. World Health Organization An integrated approach to communicable disease surveillance. Weekly Epidemiological Record 2000, 75: $1-8$

II. Centers for Disease Control and Prevention Assessment of infectious disease surveillance, Uganda 2000. MMWR 2000, 49:6879

12. Online AFP Surveillance Data, World Health Organization 2000,

13. Dean AG, Dean JA, Coulombier D and Brendel KA Epi Info, Version 6: a word processing, database, statistics program for epidemiology on microcomputers. Centers for Disease Control 1994,

14. Kleinau E Management of health information systems In: Design and implementation of health information systems (Edited by: Lippeveld $T$, Suaerborn R, Bodart C) Geneva, Switzerland, World Health Organization 2000, 176-197

15. Centers for Disease Control and Prevention Updated guidelines for evaluating public health surveillance systems: recommendations from the guidelines working group. MMWR 200I, 50(No. RR-13):

16. World Health Organization, Regional Office for Africa Protocol for the assessment of national communicable disease surveillance and epidemic preparedness and response systems. AFR/ IDS/0I.I 2000,

\section{Pre-publication history}

The pre-publication history for this paper can be accessed here:

http://www.biomedcentral.com/1471-2458/2/27/prepub 